\title{
The Parameter Space of Orbits of a Maximal Compact Subgroup Acting on a Flag Manifold
}

\author{
B. Ntatin $(D)$ \\ Department of Mathematics, Austin Peay State University, Clarksville, TN 37043, USA \\ Correspondence should be addressed to B. Ntatin; ntatinb@apsu.edu
}

Received 1 March 2019; Revised 26 April 2019; Accepted 20 May 2019; Published 1 July 2019

Academic Editor: Ali Jaballah

Copyright (C) 2019 B. Ntatin. This is an open access article distributed under the Creative Commons Attribution License, which permits unrestricted use, distribution, and reproduction in any medium, provided the original work is properly cited.

\begin{abstract}
The orbits of a real form $G$ of a complex semisimple Lie group $G^{\mathbb{C}}$ and those of the complexification $K^{\mathbb{C}}$ of its maximal compact subgroup $K$ acting on $Z=G^{\mathbb{C}} / Q$, a homogeneous, algebraic, $G^{\mathbb{C}}$-manifold, are finite. Consequently, there is an open $G$-orbit. Lower-dimensional orbits are on the boundary of the open orbit with the lowest dimensional one being closed. Induced action on the parameter space of certain compact geometric objects (cycles) related to the manifold in question has been characterized using duality relations between $G$ - and $K^{\mathbb{C}}$-orbits in the case of an open $G$-orbit and more recently lower-dimensional $G$-orbits. We show that the parameter space associated with the unique closed $G$-orbit in $Z$ agrees with that of the other orbits characterized as a certain explicitly defined universal domain.
\end{abstract}

\section{Introduction}

Let $G$ be a noncompact semisimple Lie group which is embedded in its complexification $G^{\mathbb{C}}$ and let $Q$ be a parabolic subgroup in the sense that it contains a Borel subgroup of $G^{\mathbb{C}}$, then $Z=G^{\mathbb{C}} / Q$ is a compact, homogeneous, algebraic, rational $G^{\mathbb{C}}$-flag manifold. Observe that $G$ as well as $G^{\mathbb{C}}$ acts naturally on every flag manifold $Z=G^{\mathbb{C}} / Q$. Since $G$ is semisimple, it decomposes as a product of almost simple factors $G=G_{1} \times G_{2} \times \cdots \times G_{k}$ in the sense that the map $\Pi_{i} G_{i} \longrightarrow G$ is surjective with finite kernel. This leads to a corresponding decomposition of the flag manifold $Z=$ $Z_{1} \times Z_{2} \times \cdots \times Z_{k}$ as a finite direct product with irreducible factors $Z_{i}=G_{i}^{\mathbb{C}} / Q_{i}$, where for each $i, Q_{i}=Q \cap G_{i}$ is a parabolic subgroup of the complexification $G_{i}^{\mathbb{C}}$ of the simple factors $G_{i}$. Hence a $G$-orbit (respectively, $G^{\mathbb{C}}$-orbit) in $Z$ is a product of $G_{i}$-orbits (respectively, $G_{i}^{\mathbb{C}}$-orbits) in the corresponding factors $Z_{i}$. As a consequence, when we assume in the sequel that $G$ is semisimple; it is with the understanding that the above decomposition is possible.

Let $\theta$ be a Cartan involution of $G$ (see Section 2.1), then its fixed point set $K:=G^{\theta}$ is a maximal compactly embedded subgroup of $G$. We extend $\theta$ holomorphically to $G^{\mathbb{C}}$ (and still call it $\theta$ ), then its fixed point set is the complexification
$K^{\mathbb{C}}:=\left(G^{\mathbb{C}}\right)^{\theta}$ of the maximal compact subgroup. The maximal subgroup $K$ as well as its complexification $K^{\mathbb{C}}$ also acts naturally on $Z$. In fact, $G / K$ is a negatively curved Riemannian symmetric space embedded in $G^{\mathbb{C}} / K^{\mathbb{C}}$.

In the sequel, we will follow the notation introduced in [1] or [2], and let $\operatorname{Orb}_{Z}(G)$ (resp., $\operatorname{Orb}_{Z}\left(K^{\mathbb{C}}\right)$ ) denote the set of all $G$-orbits (resp., $K^{\mathbb{C}}$-orbits) in $Z$. It is known that these sets are finite [3]. As a consequence, there is at least one open $G$-orbit since the $G$-action on $Z$ is algebraic. Indeed, all lowerdimensional $G$-orbits are found in the boundary of an open orbit with the minimal-dimensional orbit being closed. In fact there is only one closed $G$-orbit in $Z$.

There exists a duality relation between $G$-orbits and $K^{\mathbb{C}}$ orbits in the flag manifold $Z$ : let $\kappa \in \operatorname{Orb}_{Z}\left(K^{\mathbb{C}}\right)$ and $\gamma \in$ $\operatorname{Orb}_{Z}(G)$, then the pair $(\kappa, \gamma)$ is said to be a dual pair if $\kappa \cap \gamma$ is nonempty and compact. Observe that if $\gamma$ is an open $G$-orbit, then $(\kappa, \gamma)$ is a dual pair if and only if $\kappa \subset \gamma$; moreover, such a $\kappa$ is unique [3]. This duality relation has been extended to include all $G$ - and $K^{\mathbb{C}}$-orbits $[1,4-7]$. For every $\gamma \in \operatorname{Orb}_{Z}(G)$ there exists a unique $\kappa \in \operatorname{Orb}_{Z}\left(K^{\mathbb{C}}\right)$ such that $(\gamma, \kappa)$ is a dual pair and vice versa.

If $D$ is the open $G$-orbit in the flag manifold $Z$, let $C_{0}$ be the dual $K^{\mathbb{C}}$-orbit and denote by $\mathscr{C}_{q}(D)$, where $q:=\operatorname{dim}_{\mathbb{C}} C_{0}$, 
the space of all $q$-dimensional compact cycles in $D$. Observe that $C_{0}$ is compact and contained in $D$ and hence just a point in $\mathscr{C}_{q}(D)$. By associating $g \in G^{\mathbb{C}}$ to the $g$-translate $g\left(C_{0}\right)$ of $C_{0}$, the connected component $\Omega_{W}(D)$ of the set

$$
\left\{g \in G^{\mathbb{C}}: g\left(C_{0}\right) \subset D\right\}
$$

can be regarded as a family of $q$-dimensional cycles. Since $\Omega_{W}(D)$ is invariant by the right $K^{\mathbb{C}}$-action on the right, we often regard it as being in the affine homogenous space $G^{\mathbb{C}} / K^{\mathbb{C}}$.

In the present paper, we generalize this definition to any lower-dimensional $G$-orbit $\gamma \in \operatorname{Orb}_{Z}(G)$ and hence to the case of the unique closed $G$-orbit. We extend results and methods originally developed in [2], which is the author's $\mathrm{PhD}$ dissertation) for lower-dimensional orbits to the case of the unique closed orbit. For instance, Proposition 7 is implicit in [8] where it was only proven that the intersection of the base cycle with a Schubert slice is finite. Here we prove that this intersection is a single point. We give some interesting examples of Matsuki dual orbits in the Hermitian case in Section 4. This helps in Section 5 to characterize the nature of the unique closed orbit in the full flag manifold in terms of signatures of a Hermitian form (Proposition 19). In Section 6, we use known results from [1, 2, 9] together with a result about cycle ordering (Proposition 21), to prove our main result (Theorem 24) that the cycle space of the closed orbit coincides with the universal domain $\Omega_{A G}$.

\section{Preliminaries and Some Basic Results}

In this section, we discuss some basic results about semisimple Lie groups that we will need in the sequel. A survey of the details of the discussions in this section can be found in [10], [11], [12], or [13].

2.1. Cartan Decomposition and Real Forms. Recall that a Lie algebra $\mathfrak{g}$ is semisimple if and only if the killing form $B: \mathfrak{g} \times$ $\mathfrak{g} \longrightarrow \mathbb{C}$ defined by $B(x, y)=\operatorname{Tr}\left(a d_{x} a d_{y}\right)$ is nondegenerate. Note that the real Lie algebra $\mathfrak{g}$ is semisimple if and only if its complexification $\mathfrak{g} \otimes \mathbb{C} \cong \mathfrak{g} \oplus i \mathfrak{g}$ is semisimple. Here, $\mathfrak{g}$ is the fixed point set of a conjugate linear involution $\tau: \mathfrak{g}^{\mathbb{C}} \longrightarrow \mathfrak{g}^{\mathbb{C}}$. Indeed, $\tau$ defines a decomposition $\mathfrak{g}=\mathfrak{g}^{+} \oplus \mathfrak{g}^{-}$of $\mathfrak{g}$ into +1 and -1 eigenspaces. The subalgebra $\mathfrak{g}=\mathfrak{g}^{+}$is called the real form of $\mathfrak{g}$ and is characterized by the fact that its complexification is $\mathfrak{g} \otimes \mathbb{C} \cong \mathfrak{g}^{\mathbb{C}}$.

We note that $\mathfrak{g}^{\mathbb{C}}$ also has a compact real form which is the fixed point set of a compact real involution $\sigma$. Denote the +1 eigenspace of $\sigma$ by $\mathfrak{t}$, then

$$
\mathfrak{g}^{\mathbb{C}}=\mathfrak{u} \oplus i \mathfrak{u}
$$

Also, let $\mathfrak{g}^{\mathbb{C}} \cong \mathfrak{g} \oplus i \mathfrak{g}$ be the complexification of $\mathfrak{g}$. Let $\tau$ be the conjugation on $\mathfrak{g}^{\mathbb{C}}$ giving the real form $\mathfrak{g}$. Also, let $\sigma: \mathfrak{g}^{\mathbb{C}} \longrightarrow \mathfrak{g}^{\mathbb{C}}$ be the compact real involution giving the compact real form $\mathfrak{t}$. When restricted to $\mathfrak{g}$, the involution $\theta=$ $\tau \sigma=\sigma \tau$ defines an eigenspace decomposition $\mathfrak{g}^{\mathbb{C}}=\mathfrak{g}_{+}^{\mathbb{C}} \oplus \mathfrak{g}_{-}^{\mathbb{C}}$ and stabilizes both real forms $\mathfrak{g}$ and $\mathfrak{t}$. Hence we have the decompositions

$$
\begin{aligned}
\mathfrak{g} & =\mathfrak{g}_{+} \oplus \mathfrak{g}_{-}, \\
\mathfrak{u} & =\mathfrak{u}_{+} \oplus i \mathfrak{t}_{-} .
\end{aligned}
$$

Now, set $\mathfrak{f}=\mathfrak{g}_{+}$and $\mathfrak{p}=\mathfrak{g}_{-}$, then since $\theta$ agrees with $\sigma$ on $\mathfrak{g}$, it follows that $\mathfrak{f}=\mathfrak{t}_{+}$and $\mathfrak{p}=i \mathfrak{u t}$. This leads to the following decompositions:

$$
\begin{aligned}
\mathfrak{g} & =\mathfrak{f} \oplus \mathfrak{p}, \\
\mathfrak{u} & =\mathfrak{f} \oplus \mathfrak{i p} .
\end{aligned}
$$

The decomposition $\mathfrak{g}=\mathfrak{f} \oplus \mathfrak{p}$ is called a Cartan decomposition. It is an orthogonal decomposition with respect to the killing form $B$ which is negative definite on $\mathfrak{t}$ and positive definite on $\mathfrak{p}$; thus $\mathfrak{f} \subset \mathfrak{g}$ is a maximal compact subalgebra. If $G$ is the semisimple Lie group whose Lie algebra is $\mathfrak{g}$, then $\exp (\mathfrak{f})=K \subset G$ is a maximal compact subgroup of $G$.

We can lift the Cartan decomposition from the Lie algebra level to the group level.

Proposition 1. Let $G$ be a semisimple Lie group, let $\theta$ be a Cartan involution of its Lie algebra $\mathfrak{g}$, let $\mathfrak{g}=\mathfrak{f} \oplus \mathfrak{p}$ be the corresponding Cartan decomposition, and let $K$ be the analytic subgroup with Lie algebra $\mathfrak{f}$. Then,

(1) there exist a Lie algebra automorphism $\Theta$ of $G$ with differential $\theta$ which is an involution.

(2) $K=\operatorname{Fix}(\Theta)$ is the subgroup of $G$ fixed by $\Theta$.

(3) The map $K \times \mathfrak{p} \longmapsto G$ given by $(k, X) \longrightarrow k \exp (X)$ is a diffeomorphism.

(4) $K$ is closed and contains the center of $G$.

(5) If the center of $G$ is finite then $K$ is a maximal compact subgroup of $G$.

Example 2. If $\mathfrak{g}^{\mathbb{C}}=\mathfrak{g l}(3, \mathbb{C})$ then the real form $\mathfrak{g l}(3, \mathbb{R})$ is the fixed point set of the involution defined by complex conjugation. A maximal compact subgroup of the associated Lie group is $S O(3, \mathbb{R})$ or its conjugate. The Cartan involution at the Lie algebra level is given by $\theta(X)=-\bar{X}^{t}$, and at the Lie group level, it is given by $\Theta(A)=\left(\bar{A}^{-1}\right)^{t}$. The Cartan decomposition with respect to $\theta$ is

$$
\mathfrak{g l}(3, \mathbb{R})=\mathfrak{g} \mathfrak{v}(3, \mathbb{R}) \oplus \mathfrak{p}=\mathfrak{H} \oplus \mathfrak{p},
$$

where $\mathfrak{p}$ is the set of symmetric matrices with zero trace.

2.2. Iwasawa Decomposition and Parabolic Subgroups. Let $\mathfrak{g}$ be a complex semisimple Lie algebra, $\theta$ be a Cartan involution of $\mathfrak{g}$, and $\mathfrak{g}=\mathfrak{H} \oplus \mathfrak{p}$ be the corresponding Cartan decomposition. Let $\mathfrak{a}$ be a maximal abelian subspace of $\mathfrak{p}$. It follows that, for any $Y \in \mathfrak{a}, a d X$ is a self-adjoint transform of $\mathfrak{g}$ and so $\mathfrak{g}$ is an orthogonal direct sum of character spaces

$$
g_{\lambda}=\{X \in \mathfrak{g}:(a d Y) X=\lambda(Y) X, \text { for all } Y \in \mathfrak{a}\}
$$

$$
\text { for } \lambda \in \mathfrak{a}^{*} \text {. }
$$


If $\mathfrak{g}_{\lambda} \neq 0$ it is called a restricted root space and $\lambda \neq 0$ is referred to as a restricted root. Denote the set of all restricted roots by $\Sigma$.

Proposition 3. The Lie algebra $\mathfrak{g}=\mathfrak{g}_{\mathfrak{v}} \oplus \bigoplus_{\lambda \in \Sigma} \mathfrak{g}_{\lambda}$ is an orthogonal decomposition satisfying:

(1) $\left[\mathfrak{g}_{\lambda_{1}}, \mathfrak{g}_{\lambda_{2}}\right] \subseteq \mathfrak{g}_{\lambda_{1}+\lambda_{2}}$,

(2) $\theta \mathfrak{g}_{\lambda}=\mathfrak{g}_{-\lambda}$, thus if $\lambda \in \Sigma$ then $-\lambda \in \Sigma$,

(3) $\mathfrak{g}_{\mathfrak{v}}=\mathfrak{a} \oplus \mathfrak{m}$ orthogonally, where $\mathfrak{m}=Z_{\mathfrak{f}}(\mathfrak{a})$.

The dimension of $\mathfrak{a}$ is called the real rank of $\mathfrak{g}$ and if it coincides with the rank of $\mathfrak{g}$, then $\mathfrak{g}$ is a split form.

This decomposition is called the restricted root space decomposition. Choose a notion of positivity for the restricted root system $\Sigma$, and let $\Sigma^{+}$be a set of positive roots, then $\mathfrak{n}=\bigoplus_{\lambda \in \Sigma^{+}} \mathfrak{g}_{\lambda}$ is a nilpotent Lie subalgebra of $\mathfrak{g}$.

Proposition 4. For $\mathfrak{g}$ as above, $\mathfrak{g}=\mathfrak{H} \oplus \mathfrak{a} \oplus \mathfrak{n}$ is a direct sum of vector spaces, where $\mathfrak{a}$ is abelian, $\mathfrak{n}$ is nilpotent, and $\mathfrak{a} \oplus \mathfrak{n}$ is solvable.

The above decomposition is the Iwasawa decomposition.

A parabolic subgroup $Q$ of a semisimple Lie group $G$ corresponding to a parabolic subalgebra $\mathfrak{q}$ is the $G$-normalizer of $\mathfrak{q}$, i.e.,

$$
Q=\{g \in G \mid A d(g) \mathfrak{q}=\mathfrak{q}\} .
$$

A subalgebra $\mathfrak{q} \subset \mathfrak{g}$ is a parabolic subalgebra if and only if $\mathfrak{q}^{\mathbb{C}} \subset \mathfrak{g}^{\mathbb{C}}$ is a parabolic subalgebra. Again, let $\Sigma^{+} \subset \Sigma$ be the set of positive roots and consider the corresponding simple roots.

With respect to these choices, the minimal parabolic subalgebra is given by

$$
\mathfrak{b}=\mathfrak{g}_{\mathfrak{v}} \oplus \bigoplus_{\lambda \in \Sigma^{+}} \mathfrak{g}_{\lambda}
$$

The minimal parabolic subalgebra coincides with the Borel subalgebra if $\mathfrak{g}$ is a split real form. A parabolic subalgebra is one containing a minimal parabolic subalgebra, respectively, a Borel subalgebra if $\mathfrak{g}$ is split. Up to conjugation, the parabolic subalgebras of $\mathfrak{g}$ are determined by subsets $\Pi^{\prime}$ of the set of simple roots $\Pi$. For instance, let $\Delta=\Sigma^{+} \cup\{\alpha \in \Sigma$ : $\left.\alpha \in \operatorname{span}\left(\Pi^{\prime}\right)\right\}$; we get parabolic subalgebras

$$
\begin{aligned}
\mathfrak{q} & =\mathfrak{q}^{+}=\mathfrak{g}_{\mathfrak{o}} \oplus \bigoplus_{\lambda \in \Delta} \mathfrak{g}_{\lambda}, \\
\mathfrak{q}^{-} & =\mathfrak{g}_{\mathfrak{v}} \oplus \bigoplus_{\lambda \in-\Delta} \mathfrak{g}_{\lambda},
\end{aligned}
$$

which are opposite subalgebras.

By exponentiation, we obtain the corresponding parabolic subgroups at the group level. Thus $Q=Q^{+}=$ $\exp \left(q^{+}\right)$and $Q^{-}=\exp \left(q_{-}\right)$are the corresponding opposite parabolic subgroups.

Example 5. The canonical action of $G^{\mathbb{C}}=S L(3, \mathbb{C})$ on $V=\mathbb{C}^{3}$ induces actions on $\mathbb{P}(V), \mathbb{G}_{2}(V)$ and the full flag manifold $\mathbb{F}_{1,2}(V)$. The parabolic subalgebras are characterized by subsets of the set of simple roots $\Pi=\left\{\alpha_{1}, \alpha_{2}\right\}$, that is, $\emptyset,\left\{\alpha_{1}\right\},\left\{\alpha_{2}\right\}$, and $\left\{\alpha_{1}, \alpha_{2}\right\}$ corresponding to three nontrivial parabolic subgroups

$$
\begin{aligned}
Q_{\alpha_{1}} & =\left(\begin{array}{lll}
* & * & * \\
0 & * & * \\
0 & * & *
\end{array}\right), \\
Q_{\alpha_{2}} & =\left(\begin{array}{lll}
* & * & * \\
* & * & * \\
0 & 0 & *
\end{array}\right), \\
B & =Q_{\alpha_{1}, \alpha_{2}}=\left(\begin{array}{lll}
* & * & * \\
0 & * & * \\
0 & 0 & *
\end{array}\right)
\end{aligned}
$$

The corresponding opposite parabolic subgroups are therefore given by

$$
\begin{aligned}
Q_{\alpha_{1}}^{-} & =\left(\begin{array}{ccc}
* & 0 & 0 \\
* & * & * \\
* & * & *
\end{array}\right), \\
Q_{\alpha_{2}}^{-} & =\left(\begin{array}{ccc}
* & * & 0 \\
* & * & 0 \\
* & * & *
\end{array}\right), \\
B^{-} & =Q_{\alpha_{1}, \alpha_{2}}^{-}=\left(\begin{array}{lll}
* & * & 0 \\
* & * & 0 \\
* & * & *
\end{array}\right)
\end{aligned}
$$

Note that $B$ and $B^{-}$are the opposite minimal parabolic subgroup; the Borel subgroups.

We end this subsection with a statement about the Iwasawa decomposition at the Lie group level.

Proposition 6. Let $G$ be a complex semisimple Lie group and let $\mathfrak{g}=\mathfrak{H} \oplus \mathfrak{a} \oplus \mathfrak{n}$ be an Iwasawa decomposition of the Lie algebra. Also let $K, A$, and $N$ be connected subgroups of $G$ with Lie algebras $\mathfrak{f}, \mathfrak{a}$, and $\mathfrak{n}$, respectively. Then the multiplication map . : $K \times A \times N \longrightarrow G$ given be $(k, a, n) \longmapsto$ kan is a diffeomorphism and the groups $A$ and $N$ are simply connected.

2.3. The Akhiezer-Gindinkin Domain $\Omega_{A G}$. For the real symmetric space $M=G / K$, we define below a kind of neighborhood of $M$ in $Z$ which is related to the parameter space of interest. We will show that this universally defined domain agrees with the cycle space we seek. Let $\mathfrak{g}=\mathfrak{t} \oplus \mathfrak{p}$ be a Cartan decomposition of $\operatorname{Lie}(G)$ with respect to a compact real form of $\mathfrak{g}$. For this, let $\mathfrak{a} \subset \mathfrak{p}$ be an abelian subalgebra which is maximal with the condition of being contained in $\mathfrak{p}$ and let $\Phi$ be the root system on $\mathfrak{a}$. This gives an Iwasawa 
decomposition $\mathfrak{g}=\mathfrak{H}+\mathfrak{a}+\mathfrak{n}$ of $\mathfrak{g}$. Define root hyperplanes $H_{\alpha}$ as follows: for $\alpha$ a root of $\mathfrak{a}$, let

$$
H_{\alpha}:=\left\{\xi \in \mathfrak{a}: \alpha(\xi)=\frac{\pi}{2}\right\} .
$$

Now define $\omega_{A G}$ as the connected component containing the neural element $0 \in \mathfrak{a}$ of the set which is obtained from $\mathfrak{a}$ by removing all the hyperplanes $H_{\alpha}$ as $\alpha$ runs through the whole set of roots; i.e.,

$$
\begin{aligned}
\omega_{A G} & =\left(\mathfrak{a} \backslash\left(\bigcup_{\alpha \in \Pi} H_{\alpha}\right)\right)^{0} \\
& =\bigcap_{\alpha \in \Pi}\left\{\xi \in \mathfrak{a}:|\alpha(\xi)|<\frac{\pi}{2}\right\} .
\end{aligned}
$$

The domain $\Omega_{A G}$ is defined to be the open neighborhood of the Riemannian symmetric space $G / K$ in the space $\Omega$ := $G^{\mathbb{C}} / K^{\mathbb{C}}$ given by

$$
\Omega_{A G}=G \cdot \exp \left(i \omega_{A G}\right) \cdot z_{0}
$$

where $z_{0}=e \cdot K^{\mathbb{C}} \in G^{\mathbb{C}} / K^{\mathbb{C}}$ is the base point.

This domain has been called the universal domain by some authors and others have referred to it as the complex crown of the real symmetric space $G / K$ [14]. For a survey of some properties of this domain, refer to [14], [15], or [16].

\section{Schubert Varieties and Slices}

A Borel subgroup $B$ of $G^{\mathbb{C}}$ which contains the factor $A N$ of an Iwasawa decomposition $G=K A N$ of $G$ is called an IwasawaBorel subgroup of $G^{\mathbb{C}}$. For an orbit $\mathcal{O}$ of such an IwasawaBorel subgroup in $Z$, we refer to its closure in $Z$, that is, $S=$ $\operatorname{cl}(\mathcal{O}) \subset Z$, as an Iwasawa-Schubert variety, or just a Schubert variety.

Given a dual pair $(\gamma, \kappa) \in \operatorname{Orb}_{Z}(G) \times \operatorname{Orb}_{Z}\left(K^{\mathbb{C}}\right)$, an Iwasawa-Borel subgroup $B \subset G^{\mathbb{C}}$, a $B$ Schubert variety $S$, and an intersection point $z_{0} \in \kappa \cap S$, we call the $A N$-orbit $\Sigma=A N . z_{o}$ the associated Schubert slice.

Also let $c l(\kappa)$ denote the closure of the $K^{\mathbb{C}}$-orbit dual to the $G$-orbit $\gamma$. It was shown in [8] that the intersection $c l(\kappa) \cap \Sigma$ is finite. The following is a refinement of this result.

Proposition 7. For each Schubert slice $\Sigma$, the intersection $c l(\kappa) \cap \Sigma$ consists of a unique point.

Proof. Let $B$ be the Iwasawa-Borel subgroup containing the factor $A N$ of the Iwasawa decomposition $G=K A N$. For some base point $p \in Z$, let $\Sigma_{p}=A N \cdot p$, and $S=\operatorname{cl}(\mathcal{O})$ where $\mathcal{O}=B \cdot p$ is the corresponding Schubert slice and Schubert variety, respectively. Also let $\gamma$ be the Matsuki dual orbit to $\kappa$, then a dimension count shows that $\operatorname{dim}_{\mathbb{C}} \gamma=$ $\operatorname{dim}_{\mathbb{C}} \Sigma+\operatorname{dim}_{\mathbb{C}} c l(\kappa)$. As a consequence therefore of the Iwasawa decomposition $G=K A N$, it follows that $\operatorname{dim}_{\mathbb{R}} S=$ $\operatorname{dim}_{\mathbb{R}} A N . p$, hence $\Sigma=A N . p$ is open in $S$. Now, let $S \cap$ $\gamma=A_{1} \cup A_{2} \cup \cdots \cup A_{k}$ be a decomposition of $S \cap \gamma$ into its irreducible components. Every such component is $A N$ invariant and we already know that every $A N$-orbit in $\gamma$ intersects $c l(\kappa)$, so it follows that every such orbit $\Sigma=A N$. $p$ is open in $S \cap \gamma$. This shows that every component $A_{j}$, for all $j$ is a Schubert slice. This implies that the intersection $S \cap \gamma=\left\{p_{1}, \ldots, p_{k}\right\}$ is a finite set of points, where for each $j, \Sigma_{j}=\Sigma_{p_{j}}=A N \cdot p_{j}$ are the corresponding Schubert slices through $p_{j}$.

We now show that the intersection $\Sigma \cap \gamma$ is indeed a single point. Let $\Sigma$ be the slice through $p$ and suppose $\Sigma \cap c l(\kappa)=$ $\left\{p, p^{\prime}\right\}$ where $p^{\prime} \neq p$ is another point different from $p$. Since $c l(\kappa)$ is $K$-invariant, as the closure of a $K$-orbit, it follows that there exists $k \in K$ such that $k \cdot p^{\prime}=p$. Also, there exists $(a n) \epsilon$ $A N$ such that $(a n) \cdot p=p$, since $p \in \Sigma$. It therefore follows that $g=k a n \in \operatorname{Stab}_{G}(p)$, the stabilizer subgroup of the element $p$.

It was shown in [8] that the map

$$
\begin{aligned}
\alpha: \operatorname{Stab}_{K}(p) \times \operatorname{Stab}_{A N}(p) \\
\quad \longrightarrow \operatorname{Stab}_{G}(p) \text { given by }(k, a n) \longmapsto g=k a n
\end{aligned}
$$

is a diffeomorphism. Since $\gamma \cap \kappa$ is a deformation retract of $\gamma$, it follows that $\alpha$ is onto. Thus, $k \in \operatorname{Stab}_{K}(p)$, and so $k \cdot p^{\prime}=$ $p^{\prime}=p$. Consequently, $(k a n) \cdot p^{\prime}=p$.

In order to give a suitable definition of the cycle space associated with a $G$-orbit in any flag manifold $Z=G^{\mathbb{C}} / Q$, we need the following result (see [8]).

Proposition 8. Let $\gamma, \kappa) \in \operatorname{Orb}_{Z}(G) \times \operatorname{Orb}_{Z}\left(G^{\mathbb{C}}\right)$ be a dual pair and $S$ the associated Schubert variety. Then

(1) $S \cap \operatorname{cl}(\kappa) \subset \kappa \cap \gamma$.

(2) The map $K \times \operatorname{cl}(\Sigma) \longrightarrow \operatorname{cl}(\gamma)$, given by $(\kappa, z) \longmapsto k(z)$ is onto, that is, $\operatorname{K.cl}(\Sigma)=c l(\gamma)$.

Corollary 9. Given a point $p$ in the closure of a G-orbit $\gamma$, that is, $p \in \operatorname{cl}(\gamma)$, there exists some Schubert variety $S$ containing $p$.

Proof. Given an Iwasawa decomposition $G=K A N$ of $G$, let $B$ be the Iwasawa-Borel subgroup containing the factor $A N$. Let $z_{0} \in \kappa \cap \gamma$ be the base point and let $S$ be the Schubert variety through $z_{0}$, that is, $S=\operatorname{cl}\left(B . z_{0}\right)$. Also, let $\Sigma=A N . z_{0}$ be the associated Schubert slice through $z_{0}$. Now suppose $p \in c l(\gamma)$, then it follows from the above result, Proposition 8, that there exits an element $k \in K$ such that $p \in K . c l(\Sigma)$. The $K$-conjugate $K(A N) K^{-1}$ of $A N$ and the $K$-conjugate $K B K^{-1}$ of $B$ satisfy the inclusion; $K(A N) K^{-1} \subset$ $K B K^{-1}$. As a consequence, $p \in c l\left(K(A N) K^{-1} \cdot z_{0}\right)=$ $\operatorname{cl}\left(\left(K A K^{-1}\right)\left(K N K^{-1}\right) \cdot z_{0}\right)$. Set $\widetilde{A}=K(A) K^{-1}$, and $\widetilde{N}=$ $K(N) K^{-1}$, then it follows that $p \in \operatorname{cl}\left(\widetilde{A} \widetilde{N} . z_{0}\right) \subset \operatorname{cl}\left(\widetilde{B} . z_{0}\right)=$ $\widetilde{S}$, where $\widetilde{S}$ is some other Schubert variety associated with another Iwasawa decomposition $G=K \widetilde{A} \widetilde{N}$. That is, $S$ is the closure $\operatorname{cl}(\widetilde{\mathcal{O}}=\operatorname{cl}(\widetilde{B})$ of the orbit $\widetilde{\mathcal{O}}$ of another Iwasawa-Borel subgroup which contains the factor $\widetilde{A} \widetilde{N}$.

Lemma 10 ([2], see Lemma 1.7). Let $(\gamma, \kappa) \in \operatorname{Orb}_{Z}(G) \times$ $\operatorname{Orb}_{Z}\left(G^{\mathbb{C}}\right)$ be a dual pair, then $\operatorname{cl}(\kappa) \cap \operatorname{cl}(\gamma)=\kappa \cap \gamma$. 
Proof. Since $\kappa \in \operatorname{cl}(\kappa)$ and $\gamma \in \operatorname{cl}(\gamma)$, it follows that $c l(\kappa) \cap$ $c l(\gamma) \subset \kappa \cap \gamma$. Now suppose $p \in \operatorname{cl}(\kappa) \cap \operatorname{cl}(\gamma)$, then by the first part of Proposition 8, there exists some Schubert variety $S$ containing the point $p$, that is, $p \in \operatorname{cl}(\kappa) \cap S$, but this intersection by Proposition 8 is contained in $\kappa \cap \gamma$.

Proposition 11. Let $(\gamma, \kappa) \in \operatorname{Orb}_{Z}(G) \times \operatorname{Orb}_{Z}\left(G^{\mathbb{C}}\right)$ be a dual pair, then the identity element is in the interior of the set

C

$$
:=\left\{g \in G^{\mathbb{C}}: g(\kappa) \cap \gamma \text { is non-empty and compact }\right\} .
$$

Proof. It follows from Lemma 10 that $\operatorname{cl}(\kappa) \cap \operatorname{cl}(\gamma)=\kappa \cap$ $\gamma \subset \gamma$. Let $d: Z \times Z \longrightarrow \mathbb{R}^{\geq 0}$ be any distance function on $Z$. Since $Y:=c l(\kappa)$ is compact in $Z$, it follows that the distance from $Y$ to $b d(\gamma)$, the boundary of $\gamma$, is positive, that is, $d(Y, b d(\gamma))>0$. Observe that the map $\alpha: G^{\mathbb{C}} \longrightarrow \mathbb{R}^{\geq 0}$ defined by $g \longmapsto d(g(Y), b d(\gamma))$ is a continuous map. In particular, observe that since $\alpha(I d)>0$, it follows that there exists a neighborhood $U$ of the identity $I d \in G^{\mathbb{C}}$ such that, for all $g \in U, d(Y, b d(\gamma))>0$. Equivalently, for all $g \in U$, $g(Y) \cap c l(\gamma) \subset \gamma$. Now, since $\gamma$ and $\kappa$ are dual, the intersection $\gamma \cap \kappa$ is transversal; it therefore follows that the intersection $g(\kappa) \cap \gamma$ ) also remains transversal for $g$ in a neighborhood of the identity possibly smaller than $U$. Thus, the set $C$ is nonempty, and in particular, the identity $I d \in G^{\mathbb{C}}$ is and interior point of $C$.

Definition 12. Let $(\gamma, \kappa) \in \operatorname{Orb}_{Z}(G) \times \operatorname{Orb}_{Z}\left(G^{\mathbb{C}}\right)$ be a dual pair. The cycle space $C(\gamma)$ associated with a $G$-orbit $\gamma$ is defined to be the connected component containing the identity of the interior of the set

$$
\begin{aligned}
& C(\gamma) \\
& \quad=\left\{g \in G^{\mathbb{C}}: g(\kappa) \cap \gamma \text { is non-empty and compact }\right\} .
\end{aligned}
$$

Clearly, by Proposition $11 C(\gamma)$ is a nonempty open subset of $G^{\mathbb{C}}$ containing the identity element $I d \in G^{\mathbb{C}}$. This definition agrees with the definition of the cycle domain $\Omega_{W}(D)$ introduced in [17] for the case when $\gamma=D$, an open G-orbit in Z.

Remark 13. The elements of $C(\gamma)$ are clearly transformations since as a set, $C(\gamma) \subset G^{\mathbb{C}}$. Sometimes we will want to think of this set as $\mathscr{C}_{q}(Z)$, the set of $q$-dimensional cycles in $Z$. For this purpose, observe that the $G^{\mathbb{C}}$-action on $\mathscr{C}_{q}(Z)$ is algebraic; consequently, for a base cycle $C_{0}$, the orbit $G^{\mathbb{C}} \cdot C_{0}$ is identifiable with the $G^{\mathbb{C}}$-homogeneous space $G^{\mathbb{C}} / G_{C_{0}}^{\mathbb{C}}$, where $G_{C_{0}}^{\mathbb{C}}$ is the isotropy subgroup at the base cycle.

It follows therefore that $C(\gamma)$ is invariant under right multiplication by $G_{C_{0}}^{\mathbb{C}}$. As a result we may think of cycles as being in $\mathscr{C}_{q}(Z)$ by replacing $C(\gamma)$ by $C(\gamma) / G_{C_{0}}^{\mathbb{C}}$ and vice versa.

It turns out that the complex group $K^{\mathbb{C}}$ is always a subgroup of the isotropy subgroup $G_{C_{0}}^{C}$. Indeed, if $G$ is not of Hermitian type, then $K^{\mathbb{C}}$ is maximal in $G^{\mathbb{C}}$ in the sense that the only proper subgroups which contain it are finite extensions. Hence in the nonhermitian case, $G_{C_{0}}^{\mathbb{C}}$ is at most a finite extension of $K^{\mathbb{C}}$.

On the other hand, in the Hermitian case, $K^{\mathbb{C}}$ is contained in one of the parabolic subgroups $P_{ \pm}$, where $G^{\mathbb{C}} / P_{ \pm}$are the associated compact Hermitian symmetric spaces. It is possible that, in this case, $G_{C_{0}}^{\mathbb{C}}=P_{-}$or $P_{+}$. For instance, if $x_{-} \in G^{\mathbb{C}} / P_{-}$is the base point and $\gamma=G . x_{-}$is the open $G$ orbit, then the dual $K^{\mathbb{C}}$-orbit $\kappa=C_{0}$ is just the base point, and so $G_{C_{0}}^{\mathbb{C}}=P_{-}$.

In the sequel therefore, we will often view $C(\gamma)$ as being in $\mathscr{C}_{q}(Z)=G^{\mathbb{C}} / G_{C_{0}}^{\mathbb{C}}$, and either by pulling back the fibrations $G^{\mathbb{C}} / K^{\mathbb{C}} \longrightarrow G^{\mathbb{C}} / P_{ \pm}$or considering a finite cover for $K^{\mathbb{C}}$, we will regard it as being in $G^{\mathbb{C}} / K^{\mathbb{C}}$.

We will make use of some results and methods developed in $[5,6,8,9,12,13,15,17-19]$ to prove our results.

It was conjectured in [20] that the cycle space coincided with the domain $\Omega_{A G}$ in certain cases, precisely when $Z=$ $G^{\mathbb{C}} / Q$ is of nonholomorphic type. This conjecture has been shown to be true for the open $G$-orbit by using combinatorial computations involving closures of $K^{\mathbb{C}}$-orbits in $\mathrm{Z}$.

Fix an open $G$-orbit $D$ in $Z=G^{\mathbb{C}} / Q$, a base cycle $C_{0}$ in $D$ and let $\Omega:=G^{\mathbb{C}} \cdot C_{0}$ denote the corresponding $G^{\mathbb{C}}$-orbit in the cycle space $\mathscr{C}_{q}(D)$. The cycle space $\Omega_{W}(D)$ associated with open $G$-orbits in any flag manifolds $Z$ has been completely characterized [5]. Furthermore, if $\gamma \in \operatorname{Orb}_{Z}(G)$ is not open (i.e., if $\gamma$ is a lower-dimensional orbit), then the following theorem has been proved for $G$ not of Hermitian type.

Theorem 14 (see [1]). If $G$ is not of Hermitian type, then the cycle space associated with $\gamma$

$$
C(\gamma)=\Omega_{A G}
$$

for all $\gamma \in \operatorname{Orb}_{Z}(G)$.

As a note added in proof in [1], the case for when $G$ is of Hermitian type has also been considered.

Proposition 15 (see [1]). If $G$ is of Hermitian type, then either

$$
C(\gamma)=\Omega_{A G}=\mathscr{B} \times \overline{\mathscr{B}}
$$

or the base cycle $c l(\kappa)$ is $P$-invariant and $C(\gamma)$ is either $\mathscr{B}$ or its complex conjugate $\overline{\mathscr{B}}$ depending on the choice of sign.

\section{Example of Dual Orbits}

In this section, we present an example in which we determine all the orbits of a real form of a Lie group and their corresponding Matsuki duals, specifying which orbits are open and which ones are closed. This same example was considered in ([20], Example 1.5) with the purpose of understanding the Akhierzer-Gindikin domain $D / K^{\mathbb{C}}$ where $D$ is an open $G$ orbit. 
Example 16. Let $V=\mathbb{C}^{3}, G^{\mathbb{C}}=S L_{3}(\mathbb{C})$, and $G=S U(1,2)=$ $\left\{g \in G^{\mathbb{C}}: b(v, w)=b(g v, g w), v, w \in \mathbb{C}^{3}\right\} \subset G^{\mathbb{C}}$ be the subgroup of the isometry group of the Hermitian form $b$ : $\mathbb{C}^{3} \longrightarrow \mathbb{C}^{3}$ defined for $z=\left(z_{0}, z_{1}, z_{2}\right) \in \mathbb{C}^{3}$ by $b(z)=-\left|z_{0}\right|^{2}+$ $\left|z_{1}\right|^{2}+\left|z_{2}\right|^{2}$. Since multiplication by a constant has no effect in the projective space, it follows that the orbits of $S U(1,2)$ and $U(1,2)$ coincide on $\mathbb{P}^{2}(\mathbb{C})$. Consequently, there are three orbits by Witt's theorem, namely,

$$
\begin{aligned}
& \gamma_{-}=\left\{l \in \mathbb{P}\left(\mathbb{C}^{3}\right):\left.b\right|_{l}<0\right\}, \\
& \gamma_{+}=\left\{l \in \mathbb{P}\left(\mathbb{C}^{3}\right):\left.b\right|_{l}>0\right\}, \\
& \gamma_{0}=\left\{l \in \mathbb{P}\left(\mathbb{C}^{3}\right):\left.b\right|_{l}=0\right\},
\end{aligned}
$$

Now fix a Cartan decomposition of $\mathfrak{s u}(1,2)$ determined by the decomposition $V=V_{-} \oplus V_{+}$where $V_{-}=\left\{\lambda e_{1}: \lambda \epsilon\right.$ $\mathbb{C}\}$ and $V_{+}=\left\{\lambda e_{1}+\mu e_{2}: \lambda, \mu \in \mathbb{C}\right\}$. Let $K=\{g \in$ $\left.S U(1,2): g\left(V_{ \pm}\right)=V_{ \pm}\right\}$, whose complexification is given by $K^{\mathbb{C}}=\left(G L\left(V_{-}\right) \times G\left(L\left(V_{+}\right)\right) \cap G\right.$. Observe that the group $G L\left(V_{-}\right) \times G L\left(V_{+}\right)$stabilizes the decomposition $V=V_{-} \oplus V_{+}$ and so has 4 orbits in $V$, namely, $0, V_{-} \backslash\{0\}, V_{+} \backslash\{0\}$, and $\left\{\left(v_{-}, v_{+}\right): v_{-} \neq 0, v_{+} \neq o\right\}$. These orbits are all $C^{*}$-stable hence there are three $K^{\mathbb{C}}$-orbits in $\mathbb{P}\left(v_{-}, v_{+}\right)$, namely,

$$
\begin{aligned}
\kappa_{-} & =\mathbb{P}\left(V_{-}\right)=K^{\mathbb{C}} . l_{1} \cong\{p t\} \quad \text { for } p=1, q=2, \\
\kappa_{+} & =\mathbb{P}\left(V_{+}\right)=K^{\mathbb{C}} \cdot l_{2} \cong \mathbb{P}^{1}, \\
\kappa_{-+} & =\mathbb{P}\left(v_{-} \oplus v_{+}\right) \backslash\left(\mathbb{P}\left(v_{-}\right) \cup \mathbb{P}\left(v_{+}\right)\right) \cong \mathbb{C}^{2} \backslash 0 .
\end{aligned}
$$

Observe that duality is given by the fact that $\gamma_{-} \cap \mathbb{P}\left(V_{-}\right)=$ $\mathbb{P}\left(V_{-}\right)=K . l_{1}, \gamma_{+} \cap \mathbb{P}\left(V_{+}\right)=\mathbb{P}\left(V_{+}\right)=K . l_{2}$, and $\gamma_{0} \cap K . l_{3}=$ $\gamma_{0}=K . l_{3}$; consequently, $\left(\gamma_{-}, \kappa_{-}\right),\left(\gamma_{+}, \kappa_{+}\right)$, and $\left(\gamma_{-}, \kappa_{-}\right)$are dual pairs.

The action of $G^{\mathbb{C}}$ on $\mathbb{C}^{3}$ also induces an action on the full flag manifold $\mathbb{F}_{1,2,3}\left(\mathbb{C}^{3}\right)$. Let $\left\{e_{1}, e_{2}, e_{3}\right\}$ represent the standard basis for $\mathbb{C}^{3}$; define $v_{1}=e_{2}-e_{1}, v_{2}=e_{2}+e_{1}$, and $v_{3}=e_{3}$, then $\left\{v_{1}, v_{2}, v_{3}\right\}$ is still a basis for $\mathbb{C}^{3}$. The Iwasawa-Borel subgroup is the Borel subgroup that stabilizes the full flag $\mathbb{C} v_{2} \subset\left(v_{2} \oplus\right.$ $\left.v_{3}\right) \subset \mathbb{C}^{3}$. The $G$-orbits are as follows:

$$
\begin{aligned}
& \gamma_{+,++}=\left\{(l, E) \in \mathbb{F}\left(\mathbb{C}^{3}\right):\left.b\right|_{l}<0, \operatorname{sign}(b, E)=++\right\} \\
& \gamma_{+,+-}=\left\{(l, E) \in \mathbb{F}\left(\mathbb{C}^{3}\right):\left.b\right|_{l}>0, \operatorname{sign}(b, E)=+-\right\} \\
& \gamma_{-,+-}=\left\{(l, E) \in \mathbb{F}\left(\mathbb{C}^{3}\right):\left.b\right|_{l}<0, \operatorname{sign}(b, E)=+-\right\} \\
& \gamma_{0,+-}=\left\{(l, E) \in \mathbb{F}\left(\mathbb{C}^{3}\right):\left.b\right|_{l}=0, \operatorname{sign}(b, E)=+-\right\} \\
& \gamma_{+,+0}=\left\{(l, E) \in \mathbb{F}\left(\mathbb{C}^{3}\right):\left.b\right|_{l}>0, \operatorname{sign}(b, E)=+0\right\} \\
& \gamma_{0,+0}=\left\{(l, E) \in \mathbb{F}\left(\mathbb{C}^{3}\right):\left.b\right|_{l}=0, \operatorname{sign}(b, E)=+0\right\}
\end{aligned}
$$

The orbits $\gamma_{+,++}, \gamma_{+,+-}$, and $\gamma_{-,+-}$are open in $\mathbb{F}_{1,2,3}\left(\mathbb{C}^{3}\right)$. Indeed, the fibrations $\pi_{1}: \gamma_{-,+-} \longrightarrow \gamma_{-} \cong \mathbb{B}_{2}$ and $\pi_{2}: \gamma_{+,++} \longrightarrow \gamma_{++} \cong$ $\mathbb{B}_{2}$ are holomorphic with fibers equal to $\mathbb{P}^{1}$. Since the ball can be retracted to a point, these fibration are trivial, hence $\gamma_{+,++} \cong \pi^{-} 1(\mathbb{B})=\mathbb{B}_{2} \times \mathbb{P}^{1} \cong \gamma_{++,+}$. As for the orbit, $\gamma_{+,+-}$, consider the fibration $\pi: \gamma_{+,+-} \longrightarrow \gamma_{+} \cong \mathbb{P}^{2} \backslash \overline{\mathbb{B}}_{2}$, and observe that if any holomorphic function defined on $\gamma_{+,+-}$ is restricted to $\pi^{-1}\left(\mathbb{P}^{1} \cap \gamma_{+,+-}\right)$, it must be constant by the maximum principle since every point in $\gamma_{+}$can be joined by a projective line.

The orbit $\gamma_{0,+0}$ is the unique closed orbit. This orbit is totally real since $\gamma_{0,+0} \cong K / S^{1} \cong S^{3}$.

To determine the orbits of $K^{\mathbb{C}}$, observe that the orbits of the group $G L\left(V_{-}\right) \times G L\left(V_{+}\right)$coincide with the $K^{\mathbb{C}}$-orbits in the full flag manifold $\mathbb{F}\left(\mathbb{C}^{3}\right)$. These orbits are specified by considering dimensions $\operatorname{dim}\left(V_{-} \cap l\right), \operatorname{dim}\left(V_{-} \cap l\right), \operatorname{dim}\left(V_{-} \cap E\right)$, and $\operatorname{dim}\left(V_{+} \cap E\right)$, where $l \subset E \subset \mathbb{C}^{3}$ is a full flag. Thus for

$$
\begin{aligned}
& \operatorname{dim}\left(V_{+} \cap E\right)=2, \\
& \text { let } z_{1}=\left[\mathbb{C} e_{2} \subset V_{+}\right] \text {be the base point, } \\
& \text { then } \kappa_{+,++}=K^{\mathbb{C}} \cdot z_{1}=\left\{l \subset V_{+}\right\} \cong \mathbb{P}\left(V_{+}\right) . \\
& \operatorname{dim}\left(V_{+} \cap l\right)=1=\operatorname{dim}\left(V_{-} \cap E\right), \\
& \operatorname{let} z_{2}=\left[\mathbb{C} e_{2} \subset V_{-} \oplus \mathbb{C} e_{2}\right] \text { be the base point, } \\
& \text { then } \kappa_{+,-+}=K^{\mathbb{C}} \cdot z_{2}=\left\{l \subset V_{-} \oplus l: l \in V_{+}\right\} \cong \mathbb{P}\left(V_{+}\right) . \\
& \operatorname{dim}\left(V_{+} \cap l\right)=1, \\
& \operatorname{let} z_{3}=\left[V_{-} \subset V_{-} \oplus \mathbb{C} e_{2}\right] \text { be the base point, } \\
& \text { then } \kappa_{-,-+}=K^{\mathbb{C}} \cdot z_{3} \cong \mathbb{P}\left(V_{+}\right) . \\
& \operatorname{dim}\left(V_{+} \cap l\right)=0=\operatorname{dim}\left(V_{+} \cap l\right), \text { and } \operatorname{dim}\left(V_{-} \cap E\right) \\
& \quad=1,
\end{aligned}
$$

let $z_{4}=\left[\mathbb{C} v_{1} \subset \mathbb{C} e_{1} \oplus \mathbb{C} e_{2}\right]$ be the base point,

$$
\begin{aligned}
& \text { then } \kappa_{0,-+}=K^{\mathbb{C}} . z_{4} \\
& \quad=\left\{\phi(l) \subset V_{+} \oplus \phi(l): \phi: V_{-} \longrightarrow V_{+} \text {is linear }\right\}
\end{aligned}
$$

$\operatorname{dim}\left(V_{+} \cap l\right)=1$,

$\operatorname{dim}\left(V_{-} \cap E\right)=0$,

let $z_{5}=\left[\mathbb{C} v_{3} \subset \mathbb{C} v_{1} \oplus \mathbb{C}_{2}\right]$ be the base point,

$$
\begin{aligned}
& \text { then } \kappa_{+, 0+}=K^{\mathbb{C}} \cdot z_{5} \\
& =\left\{\operatorname{ker}(\phi l) \subset \phi(l): \phi: V_{-} \longrightarrow V_{+} \text {is linear }\right\} \\
& \cong \mathbb{C}^{2} \backslash\{0\} .
\end{aligned}
$$$$
\operatorname{dim}\left(V_{+} \cap l\right)=0=\operatorname{dim}\left(V_{+} \cap l\right)=\text { and } \operatorname{dim}\left(V_{-} \cap E\right)
$$$$
=0 \text {, }
$$

let $z_{6}=\left[\mathbb{C} v_{1} \subset \mathbb{C} v_{1} \oplus \mathbb{C} v_{2}\right]$ be the base point, then $\kappa_{0,0+}=K^{\mathbb{C}} \cdot z_{6} \cong \mathbb{Q}_{3}$. 
The three orbits $\kappa_{+,++}, \kappa_{+,-+}$, and $\kappa_{-,-+}$are the $K^{\mathbb{C}}$-orbits which correspond via Matsuki duality to the three open $G$ orbits $\gamma_{+,++}, \gamma_{+,-+}$, and $\gamma_{-,-+}$, respectively, listed above. Thus, $\left(\kappa_{+,++}, \gamma_{+,++}\right),\left(\kappa_{+,-+}, \gamma_{+,-+}\right)$, and $\left(\kappa_{-,-+}, \gamma_{-,-+}\right)$are Matsuki dual pairs.

Observe that $\kappa_{0,0+}$ is the unique open $K^{\mathbb{C}}$-orbit in $\mathbb{F}_{1,2}\left(\mathbb{C}^{3}\right)$

\section{Nature of the Unique Closed G-Orbit}

In this section, we will mostly focus our attention in particular on the unique closed $G$-orbit in $Z$. First observe that when $\gamma=\gamma_{c l} \in \operatorname{Orb}_{Z}(G)$ is closed, the dual $K^{\mathbb{C}}$-orbit $\kappa_{o p}$ is open. Thus, duality in this case becomes the statement that $\kappa_{o p} \supset \gamma_{c l}$; i.e.,

$$
C\left(\gamma_{c l}\right)=\left\{g \in G^{\mathbb{C}}: g\left(\kappa_{o p}\right) \supset \gamma_{c l}\right\} .
$$

The following facts about the closed $G$-orbit are mostly a reformulation of Corollary 3.2 in [3] emphasizing the fact that the closed $G$-orbit, $\gamma_{c l}$, is unique.

As usual, let $G$ be a real form of a complex semisimple Lie group $G^{\mathbb{C}}$ and $Z=G^{\mathbb{C}} / Q$ a complex flag manifold. Let $z_{0} \in Z$ be the base point, then the following statements about the closed orbit $\gamma_{c l}=G . z_{0}$ are equivalent: $\gamma_{c l}$ is unique, contained in the closure of every $G$-orbit in $Z$, contained in every $G$ stable closed subset of $Z$, and is the lowest-dimensional $G$ orbit in $Z$. Furthermore, some maximal compact subgroup and hence every maximal compact subgroup of $G$, acts transitively on $\gamma_{c l}$ [21].

Proposition 17. Let $z \in \gamma_{c l}$, the closed G-orbit in $Z=G^{\mathbb{C}} / B$, then there is an Iwasawa-Borel subgroup $B \in G^{\mathbb{C}}$ such that $B=B_{z}$.

Proof. Let $G=K A N$ be an Iwasawa decomposition of the real group $G$, then the factor $A N$ is a simply connected algebraic group. Since its maximal compact subgroup is trivial, it follows that an $A N$-orbit that is compact most is a point. Consequently, the minimal $A N$-orbit in $\gamma_{c l}$ is a single point $z$ with the $B$-isotropy at $z$ the Borel group containing the factor $A N$. Since the maximal compact subgroup $K$ acts transitively on $\gamma_{c l}$ it follows that to every point $z \in \gamma_{c l}$ we can associate an Iwasawa-Borel subgroup; the $B$-isotropy group at $z$.

The unique closed $G$-orbit in the full flag manifold $G^{\mathbb{C}} / B$ parameterizes the set of Iwasawa-Borel subgroups of $G^{\mathbb{C}}$. Indeed, every Iwasawa-Borel subgroup stabilizes a point in the closed G-orbit.

Example 18. Let us end this section with a characterization of the closed orbit of the group $G=S U(p, q)$ acting as a real form of $G^{\mathbb{C}}=S L_{n}(\mathbb{C})$ on the full flag manifold $G^{\mathbb{C}} / B$. Actually, $G$ is the subgroup of the isometry group of the Hermitian form $h_{p, q}$ defined for $z=\left(z_{1}, \ldots, z_{p}, z_{p+1}, \ldots, z_{p+q}\right) \in \mathbb{C}^{p+q}$ by

$$
h_{p, q}(z)=\left|z_{1}\right|^{2}+\cdots+\left|z_{p}\right|^{2}-\left|z_{p+1}\right|^{2}-\cdots-\left|z_{p+q}\right|,
$$

that is, $G=\operatorname{Isom}\left(h_{p, q}\right) \cap G^{\mathbb{C}}$. Consequently the orbits of $G$ are determined by the signatures of $h_{p, q}$, with $p$ positive eigenvalues and $q$ negative eigenvalues. We give a characterization of the closed $G$-orbit in the flag manifold $G^{\mathbb{C}} / B$ below.

Proposition 19. The unique closed $G$-orbit in $G^{\mathbb{C}} / B$ is the set of all maximally isotropic full flags.

Proof. The dimensions of the null spaces, i.e., the maximally isotropic subspaces, called the Witt index is a $G$-invariant. So let $\left(n_{i}^{-}, n_{i}^{+}, n_{i}^{0}\right)$ denote the signature of the Hermitian form $h$ restricted to the subspace $\Lambda_{i}$, where $n_{i}^{0}$ denotes the dimension of the degeneracy. It follows that the maximal isotropic flags are precisely the flags with signature: $\left(0,0, n_{i}^{0}\right)$ for all $\Lambda_{i}$, $1 \leq n_{i}^{0} \leq q ;\left(n_{i}^{-}, 0, q\right)$, for all $\Lambda_{q+i}, 1 \leq n_{i}^{-} \leq p-q$; and $\left(p-q+n_{i}^{+}, n_{i}^{+}, q-n_{i}^{+}\right)$for all $\Lambda_{p+i}, 1 \leq n_{i}^{+} \leq q$. Given two such flags, there exists an element of $G$ taking one to the other. This follows by Witt's theorem, and consequently, $G$ acts transitively on the set of all these flags.

\section{Cycle Space of the Closed G-Orbit}

As usual, let $B \subset G^{\mathbb{C}}$ denote an Iwasawa-Borel subgroup. Let $\Omega:=G^{\mathbb{C}} / K^{\mathbb{C}}$ and $x_{0}=e K^{\mathbb{C}}$ be the base point, then $B \cdot x_{0}$ is open in $\Omega$. The complement of the open $B$-orbit in $\Omega$ is therefore a finite union of complex $B$-invariant hypersurfaces. Let $H$ be such a hypersurface, then the family $\{g H\}_{g \in G}$ of $G^{-}$ translates of $H$ is the same as $\{k H\}_{k \in K}$ since $H$ is $B$-invariant and $B$ contains the factor $A N$ of an Iwasawa decomposition of $G$. Now define the $G$-invariant domain

$$
\Omega_{H}:=\left(\Omega \backslash \bigcup_{g \in G}(g H)\right)^{0}=\left(\Omega \backslash \bigcup_{k \in K}(k H)\right)^{0}
$$

as the connected component containing the base point $x_{0}$ in $\Omega$.

We recall that the Iwasawa-Borel subgroup $B$ acts on the symmetric spaces $X_{ \pm}=G^{\mathbb{C}} / P_{ \pm}$.

Since $X_{ \pm}$are simply connected, irreducible compact Hermitian symmetric spaces, they have second Betti number equal to 1 . As a consequence, there is a unique $B$-invariant hypersurface in the complement of $\mathbb{B}$.

It is crucial in the characterization of the cycle space (see [1] or [2]) if the $B$-invariant hypersurface $H$ in the complement of the cycle space $C(\gamma)$ is a lift of this hypersurface.

Theorem 20 (see $[1,2])$. If the maximal B-invariant hypersurface $H$ in the complement of $C(\gamma)$ is not a lift, then

$$
\Omega_{A G}=C(\gamma)=\Omega_{H} .
$$

We will now consider the cycle space associated with the unique closed $G$-orbit $\gamma_{c l}$ for which its Matsuki dual $K^{\mathbb{C}}$-orbit $\kappa_{o p}$ is open in $Z$. Here the condition for duality reduces to the requirement that $\gamma_{c l} \subset \kappa_{o p}$ as mentioned earlier. Recall that the lower-dimensional $G$-orbits belong in the closure of the open orbit and so we may define a partial ordering of these orbits thus: for $G$-orbits $\mathcal{O}_{1}, \mathcal{O}_{2}, \mathcal{O}_{3}, \ldots$, we will write $\mathcal{O}_{1}<$ $\mathcal{O}_{2}<\mathcal{O}_{3}<\cdots$ if $\mathscr{O}_{2} \subset \operatorname{cl}\left(\mathcal{O}_{1}\right), \mathscr{O}_{3} \subset \operatorname{cl}\left(\mathcal{O}_{2}\right), \ldots$ We will need the following result about orbit orderings. 
Proposition 21. Suppose the orbit pairs $\left(\gamma_{1}, \kappa_{1}\right),\left(\gamma_{2}, \kappa_{2}\right), \ldots \in$ $\operatorname{Orb}_{Z}(G) \times \operatorname{Orb}_{Z}\left(K^{\mathbb{C}}\right)$ are dual pairs, then $\gamma_{1}<\gamma_{2}<\cdots$ if and only if $\kappa_{1}<\kappa_{2}<\cdots$.

Proof. Let $\phi_{t}$ be the gradient flow of the norm of the moment map introduced in $[4,22]$, and suppose that $\gamma_{1}<\gamma_{2}<\cdots$. It follows by definition of duality that $\gamma_{i} \cap \kappa_{i} \neq 0$ for each $i=1,2, \ldots$. This flow is invariant with respect to $K$ and is tangent to both the $G$-orbits and the $K^{\mathbb{C}}$-orbits.

So, for $p \in \gamma_{j+1} \cap \kappa_{j}$, suppose $q=\lim _{t \rightarrow \infty} \phi_{t}(p)$ belongs to $\gamma_{j} \cap \kappa_{j}$ in the sense that given any neighborhood of $q$ there exists an increasing unbounded sequence $\left\{t_{n}\right\}_{n}^{\infty} \subset \mathbb{R}$ such that $\phi_{t_{n}}(p)$ belongs to this neighborhood for every $n$. Since $c l\left(\kappa_{j}\right)$ is invariant under the flow, it follows that $\kappa_{j}=K^{\mathbb{C}} \cdot q \subset$ $c l\left(\kappa_{j+1}\right)$. By hypothesis, $\gamma_{i} \neq \gamma_{j}$ for $i \neq j$. Consequently, $\kappa_{j} \subset$ $c l\left(\kappa_{j+1}\right) \backslash \kappa_{j+1}$, that is $\kappa_{j}<\kappa_{j+1}$.

Since the flow is also $G$-invariant, we may start with $G$ orbits and repeat the argument in a similar fashion to prove the converse.

Again, let $\kappa_{o p}$ denote the open $K^{\mathbb{C}}$-orbit whose Matsuki dual is the unique closed $G$-orbit $\gamma_{c l}$. Let

$$
b d\left(\kappa_{o p}\right)=Z \backslash \kappa_{o p}=C_{1} \cup C_{2} \cup \cdots \cup C_{n}
$$

be the boundary of $\kappa_{o p}$ decomposed as a union of irreducible components. Observe that, for $j=1,2, \ldots, n, C_{j}$ contains a Zariski open $K^{\mathbb{C}}$-orbit, $\kappa_{j}$, which is dense in $C_{j}$, i.e., $\operatorname{cl}\left(\kappa_{j}\right)=$ $C_{j}$ for each $j$. Let $\gamma_{j}$ be the corresponding Matsuki dual $G$ orbit.

Corollary 22. For $\gamma_{j}$ as above for each $j$, it follows that $c l\left(\gamma_{j}\right)=$ $\gamma_{j} \cup \gamma_{c l}$, a disjoint union.

Proof. If $\gamma$ is a $G$-orbit contained in $\operatorname{cl}\left(\gamma_{j}\right) \backslash \gamma_{j}$ then its dual $K^{\mathbb{C}}$-orbit satisfies $\kappa_{j} \subset c l\left(\kappa_{j}\right) \backslash \kappa$, i.e., $\kappa_{j}<\kappa$. The only $K^{\mathbb{C}}$ orbit with this property is the one dual to the G-orbit $\gamma$ which is $\kappa=\kappa_{o p}$, the open $K^{\mathbb{C}}$-orbit. Hence $\gamma=\gamma_{c l}$ is the unique closed $G$-orbit.

Now, observe that if $g \in b d\left(C \gamma_{j}\right)$ for some $j$, then $g\left(c l\left(\kappa_{j}\right)\right) \cap b d\left(\gamma_{j}\right) \neq \emptyset$. Consequently, $g\left(c l\left(\kappa_{j}\right)\right) \cap \gamma_{c l} \neq \emptyset$. This implies in particular that $g \notin C\left(\gamma_{c l}\right)$, and we have proved the following.

Corollary 23. For $\gamma_{j}$ as above,

$$
C\left(\gamma_{c l}\right) \subset C\left(\gamma_{j}\right), \text { for each } j
$$

Theorem 24. Let $\gamma_{c l}$ be the unique closed $G$ orbit in $Z=G^{\mathbb{C}} / Q$, then it follows that

$$
C\left(\gamma_{c l}\right)=\Omega_{A G}
$$

A version of this theorem was originally proved in [2], and other versions have also appeared in $[1,15]$.

For the proof of this result, we will need the following [9]: as $\gamma$ runs through the whole set $\operatorname{Orb}_{Z}(G)$ and $Q$ runs over the set of all parabolic subgroups of $G^{\mathbb{C}}$, the intersection of all the cycle spaces $C(\gamma)$ is the same as the intersection of all the cycle spaces $C\left(\gamma_{o p}\right)$ for all open $G$-orbits in $G^{\mathbb{C}} / B$, where $B$ is a Borel subgroup of $G^{\mathbb{C}}$. That is, one has the following.

Lemma 25 (see [9]).

$$
\bigcap_{\substack{\gamma \in O r b_{Z}(G) \\ Z=G^{\mathbb{C}} / Q}} C(\gamma)=\bigcap_{\substack{\gamma=\gamma_{o p} \\ Z=G^{\mathbb{C}} / B}} C(\gamma) .
$$

Corollary 26. If $G$ is of Hermitian type and $\gamma \in \operatorname{Orb}_{Z}(G)$ is open then

$$
\bigcap_{\substack{\gamma=\gamma_{o p} \\ Z=G^{\mathbb{C}} / B}} C(\gamma)=\Omega_{A G} .
$$

Proof. We know that, in the Hermitian case, the domain $\Omega_{A G}$ coincides with $\mathbb{B} \times \overline{\mathbb{B}}$ naturally embedded in $G^{\mathbb{C}} / K^{\mathbb{C}}$ [23]. Also, recall that, in this case, $K^{\mathbb{C}}$ is properly contained in one of the opposite parabolic subgroups $P_{+}$or $P_{-}$, where $X_{+}=G^{\mathbb{C}}$ and $X_{-}=G^{\mathbb{C}}$ are the associated compact Hermitian symmetric spaces (for convenience, we will just write $X_{ \pm}$). Let $x_{ \pm}=e . P_{ \pm}$be the neutral point in $X_{ \pm}$, then $X_{ \pm}$are bounded symmetric spaces realizable as $G$-orbits. Let us denote $G . x_{+}$ by $\mathbb{B}$ and $G . x_{-}$by its dual $\overline{\mathbb{B}}$. The pull-back of these orbits by the fibrations $\pi_{ \pm}: G^{\mathbb{C}} / K^{\mathbb{C}} \longrightarrow G^{\mathbb{C}} / P_{ \pm}$gives

$$
\pi_{-}^{-1}(\mathbb{B}) \cap \pi_{+}^{-1}(\bar{B})=\mathbb{B} \times \overline{\mathbb{B}}
$$

which is embedded in $G^{\mathbb{C}} / K^{\mathbb{C}}$ as the domain $\Omega_{A G}$.

Now the following result can be deduced from results in $[5,8]$ and Lemma 25.

Corollary 27. For any $\gamma \in \operatorname{Orb}_{Z}(G)$,

$$
\bigcap_{\substack{\gamma=\gamma_{o p} \\ Z=G^{C} / B}} C(\gamma)=\Omega_{A G} \subset C(\gamma) .
$$

Proof of Theorem 24. First of all, assume that, for all $j, C\left(\gamma_{j}\right)=$ $\pi_{-}^{-1}(\mathbb{B})$ or $\pi_{+}^{-1}(\overline{\mathbb{B}})$. This implies that, for all $j, c l\left(\kappa_{j}\right)$ is $P_{ \pm^{-}}$ invariant. In particular, this would imply that $\kappa_{o p}$ is $P_{ \pm}^{-}$ invariant.

Observe that the open $K^{\mathbb{C}}$-orbit, $\kappa_{o p}$, cannot be $P_{ \pm}$invariant. Indeed, if this were the case, then $C\left(\gamma_{c l}\right) \subset \kappa_{o p}$ would be $P_{ \pm}$-invariant as well. Since $\Omega_{A G} \subset C\left(\gamma_{c l}\right)$, it would imply that $\bar{P}_{ \pm} . \Omega_{A G} \subset \Omega_{A G}$.

Since the action of $G^{\mathbb{C}} \supset P_{ \pm}$is algebraic, it follows that the $P_{ \pm}$-orbit of a generic point in $\Omega_{A G}$ is open in $\Omega$. That is, if $\kappa_{o p}$ were $P_{ \pm}$-invariant, it would follow that $C\left(\gamma_{c l}\right)$ would contain an open subset of $\Omega$. This is contrary to the fact that the complement of $C\left(\gamma_{c l}\right)$ is a union of $B$ invariant hypersurfaces, thus nonempty and $G$-invariant. This proves the result in this case since for all $j$, it follows from Corollary 27 that

$$
\Omega_{A G} \subset C\left(\gamma_{c l}\right) \subset C\left(\gamma_{j}\right) .
$$

It also follows that if for some $j, C\left(\gamma_{j}\right)=\Omega_{A G}$ then we are done. 
Furthermore, if we are in the Hermitian case and for $j \neq i$, say, we have that $C\left(\gamma_{j}\right)=\pi_{-}^{-1}(\mathbb{B})$ and $C\left(\gamma_{i}\right)=\pi_{+}^{-1}(\overline{\mathbb{B}})$, then clearly, $C\left(\gamma_{j}\right) \cap C\left(\gamma_{i}\right)=\mathbb{B} \times \overline{\mathbb{B}}=\Omega_{A G}$, and we are done as well.

\section{Data Availability}

No data were used to support this study.

\section{Disclosure}

The author discloses that some parts of this work are a direct follow-up to the author's $\mathrm{PhD}$ thesis at the Ruhr Universität, Bochum, Germany.

\section{Conflicts of Interest}

The author declares that there are no conflicts of interest regarding the publication of this paper.

\section{Acknowledgments}

The author would like to thank Prof. Dr. Dr. h.c.mult. Alan T. Huckleberry for initially introducing him to the general area of Lie group theory and Global Analysis and this topic in particular.

\section{References}

[1] A. Huckleberry and B. Ntatin, "Cycles in G-orbits in GC-flag manifolds," Manuscripta Mathematica, vol. 112, no. 4, pp. 433440, 2003.

[2] B. Ntatin, On the cycle spaces associated to orbits of semisimple lie groups [doctoral thesis], Ruhr-Universität Bochum, Bochum, Germany, 2004.

[3] J. A. Wolf, "Real groups transitive on complex flag manifolds," Proceedings of the American Mathematical Society, vol. 129, no. 8, pp. 2483-2487, 2001.

[4] R. Bremigan and J. Lorch, "Orbit duality for flag manifolds," Manuscripta Mathematica, vol. 109, no. 2, pp. 233-261, 2002.

[5] G. Fels and A. Huckleberry, "Characterization of cycle domains via Kobayashi hyperbolicity," Bulletin de la Société Mathématique de France, vol. 133, no. 1, pp. 121-144, 2004.

[6] T. Matsuki, "The orbits of affine symmetric spaces under the action of minimal parabolic subgroups," Journal of the Mathematical Society of Japan, vol. 31, no. 2, pp. 331-357, 1979.

[7] I. Mirković, T. Uzawa, and K. Vilonen, "Matsuki correspondence for sheaves," Inventiones Mathematicae, vol. 109, no. 2, pp. 231-245, 1992.

[8] A. T. Huckleberry and J. A. Wolf, "Schubert varieties and cycle spaces," Duke Mathematical Journal, vol. 120, no. 2, pp. 229-249, 2003.

[9] S. Gindikin and T. Matsuki, "Stein extensions of Riemannian symmetric spaces and dualities of orbits on flag manifolds," Transformation Groups, vol. 8, no. 4, pp. 333-376, 2003.

[10] A. Knapp, Liegroups beyond an Introduction, vol. 140 of Progress in Mathematics, Birkhäuser Boston Inc, Boston, Mass, USA, 2nd edition, 2002.
[11] A. L. Onishchik, Lectures on Real Semisimple Lie Algebras and Their Representations, ESI Lectures in Mathematics and Physics, European Mathematical Society (EMS), Zürich, Switzerland, 2004.

[12] S. Helgason, Differential Geometry and Symmetric Spaces, Academic Press, New York, NY, USA, 1962.

[13] S. Helgason, Differential Geometry, Lie Groups, and Symmetric Spaces, Academic Press, New York, NY, USA, 1978.

[14] D. N. Akhiezer and S. G. Gindikin, "On Stein extensions of real symmetric spaces," Mathematische Annalen, vol. 286, no. 1-3, pp. $1-12,1990$.

[15] G. Fels, A. Huckleberry, and J. A. Wolf, Cycle Spaces of Flag Domains: A Complex Geometric Viewpoint, vol. 245 of Progress in Mathematics, Birkhäuser, Boston, Mass, USA, 2006.

[16] R. J. Crittenden, "Minimum and conjugate points in symmetric spaces," Canadian Journal of Mathematics, vol. 14, pp. 320-328, 1969.

[17] R. O. Wells Jr. and J. A. Wolf, "Poincare series and automorphic cohomology on flag domains," Annals of Mathematics: Second Series, vol. 105, no. 3, pp. 397-448, 1977.

[18] D. Barlet and V. Koziarz, "Fonctions holomorphes sur l'espace des cycles: la methode d'intersection," Mathematical Research Letters, vol. 7, no. 5-6, pp. 537-549, 2000.

[19] A. Huckleberry, "On certain domains in cycle spaces of flag manifolds," Mathematische Annalen, vol. 323, no. 4, pp. 797-810, 2002.

[20] T. Matsuki, "Equivalence of domains arising from duality of orbits on flag manifolds," Transactions of the American Mathematical Society, vol. 358, no. 5, pp. 2217-2245, 2006.

[21] D. Montgomery, "Simply connected homogeneous spaces," Proceedings of the American Mathematical Society, vol. 1, pp. 467-469, 1950.

[22] F. Kirwan, Cohomology of Quotients in Symplectic and Algebraic Geometry, Mathematical Notes 31, Princeton University Press, Princeton, NJ, USA, 1984.

[23] D. Burns, S. Halverscheid, and R. Hind, "The geometry of Grauert tubes and complexification of symmetric spaces," Duke Mathematical Journal, vol. 118, no. 3, pp. 465-491, 2003. 


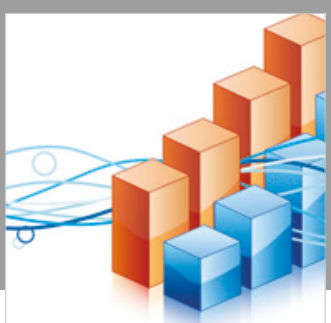

Advances in

Operations Research

\section{-n-m}
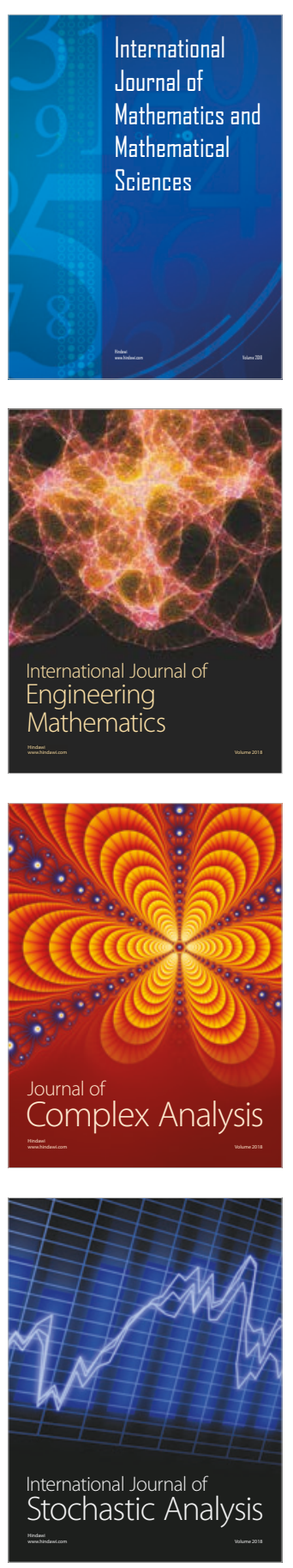
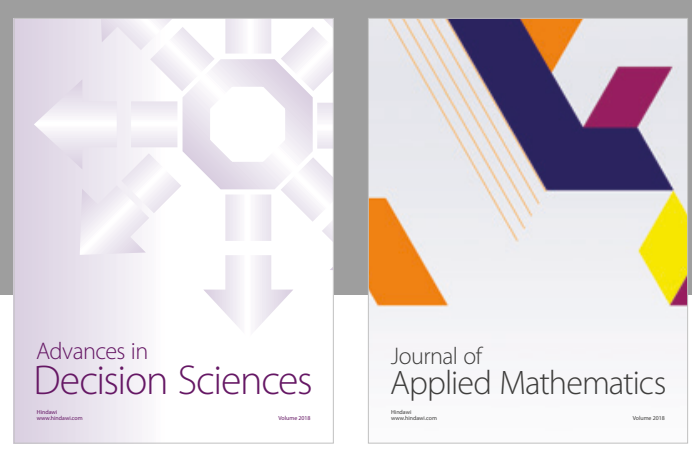

Journal of

Applied Mathematics
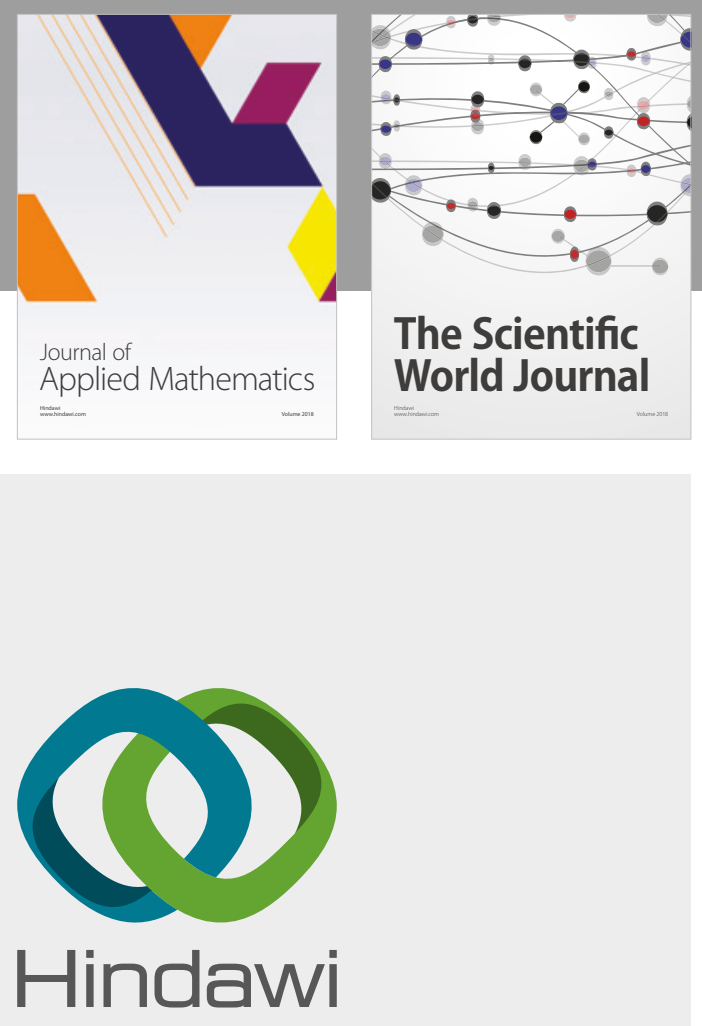

Submit your manuscripts at

www.hindawi.com

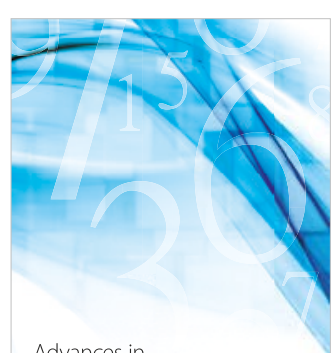

Advances in
Numerical Analysis
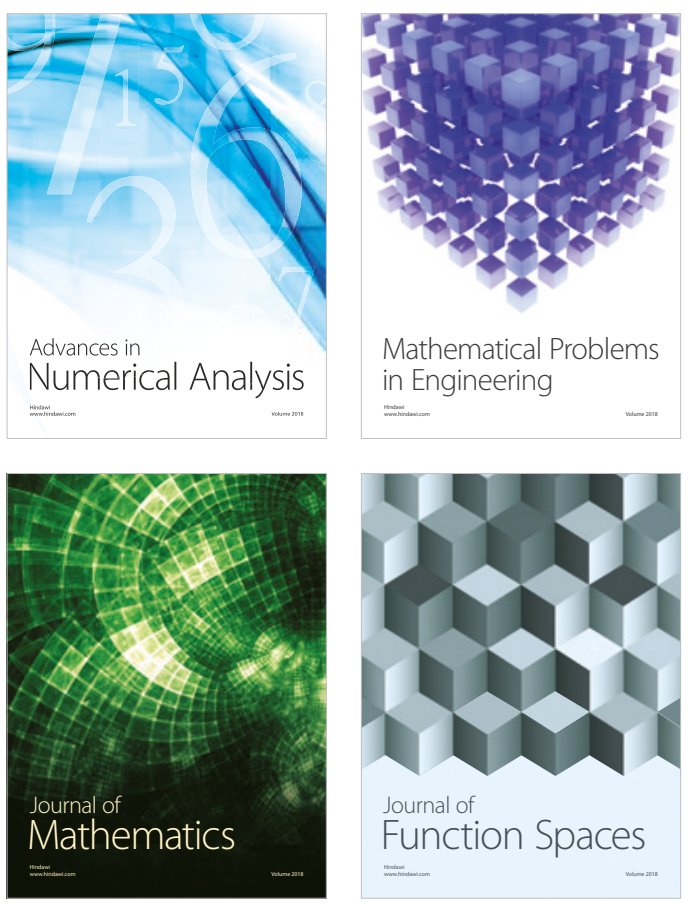

Mathematical Problems in Engineering

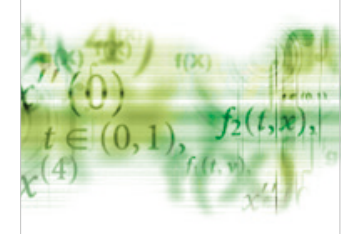

International Journal of

Differential Equations

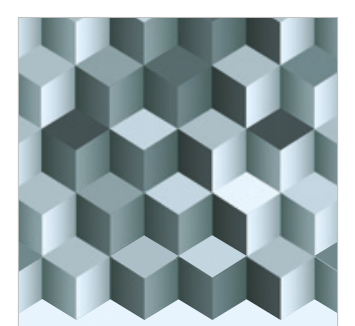

Journal of

Function Spaces
The Scientific

World Journal

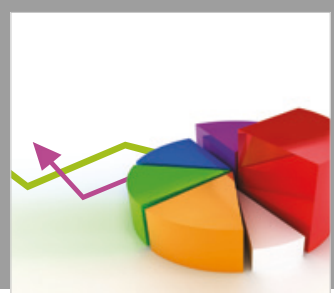

Journal of

Probability and Statistics
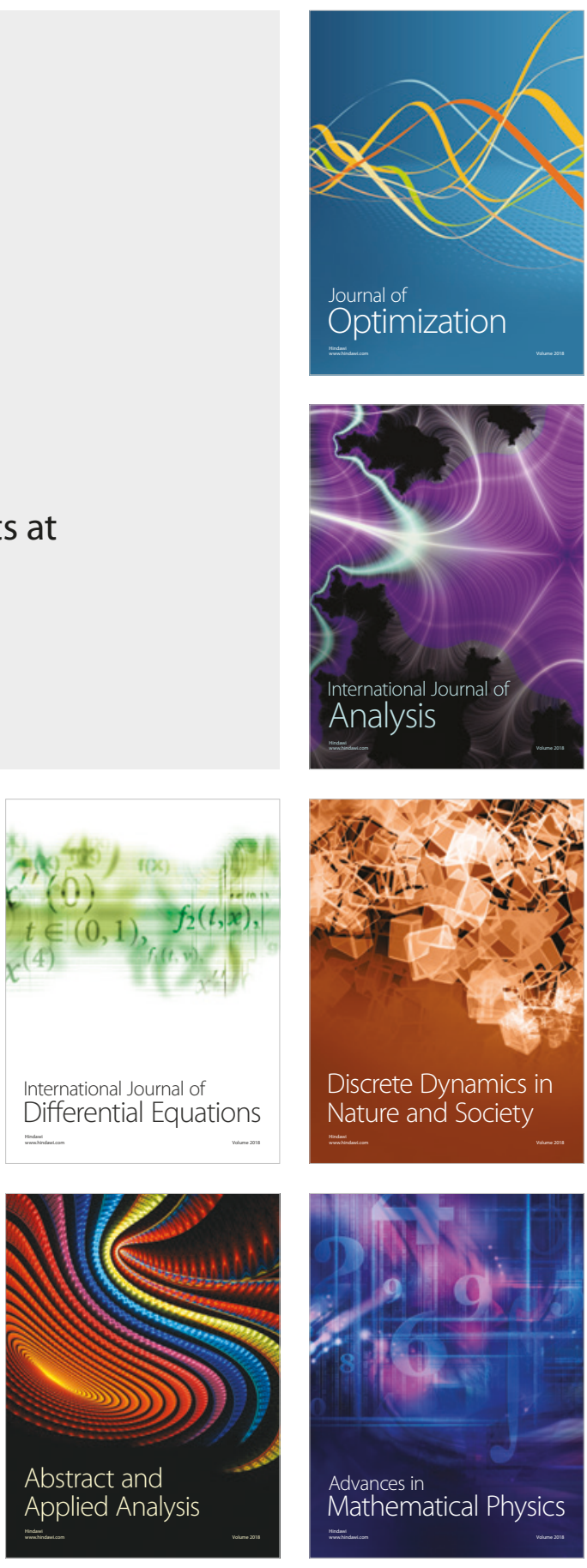